\title{
LA VALEUR DE LA STIMULATION LUMINEUSE INTERMITTENTE EN TANT QU'ÉPREUVE D'ACTIVATION DANS L'ÉLECTROENCÉ- PHALOGRAPHIE DE L'ENFANT
}

\author{
P. I.AGET *
}

L'électroencéphalographie est devenue depuis un peu plus d'une dizaine d'années une technique journellement employée tant pour la recherche expérimentale que pour l'investigation clinique. Comme telle, elle a fourni de très nombreux renseignements d'une valeur indiscutable, voire complètement bouleversé les cadres nosologiques et diagnostiques de certaines affections neurologiques comme l'épilepsie. Je ne rappellerai que pour mémoire les précisions qu'elle peut apporter dans le diagnostic différentiel des crises convulsives, la détection et la localisation des abscès et tumeurs cérébrales, l'apréciation de la valeur objective d'une thérapeutique anticonvulsivante.

On doit cependant convenir que l'essentiel à la fois des recherches et de nos connaissances portent sur l'activité électrique corticale de l'adulte et que l'électroencéphalogramme de l'enfant reste, à bien des égards, moins connu et en conséquence moins pratiqué; il n'est que de feuilleter les revues spécialisées pour remarques le petit nombre de mémoires qui y sont consacré. Les raisons de cette relative carence sont nombreuses, et certaines presque évidentes. Elles relèvent à la fois des techniques d'examen et de l'interprétation des résultats.

L'enfant n'est, en effet, pas un sujet dont il est facile d'enregistrer l'électroencéphalogramme: son indocilité, sa pusillanimité, son impatience, en font à l'état de veille un sujet remuant, instable, souvent opposant et ceci parfois avec violence. Ces faits de constatation courante expliquent et justifient l'utilisation de méthodes génératrices de tranquillité, c'est à dire la pratique de l'EEG de sommeil, sommeil en général provoqué par les barbituriques à effet rapide type Nembutal ou Seconal. Méthodes justifiables donc, mais qui ne sont pas toujours sans danger chez les enfants très jeunes et à l'origine de fréquentes erreurs.

Enfin, dans les cas les p'us favorables, lorsqu'il a été possible d'obtenir des enregistrements convenables de veille, il est indiscutable que leur

Conferência feita no Serviço de Neurologia da Escola Paulista de Medicina, em 19 de novembro de 1953.

* Do Lab. de Physiologie Générale (Faculté des Sciences - Université de Paris). Professor contratado para a Cátedra de Fisiologia da Faculdade de Medicina de Ribeirão Preto (Estado de São Paulo). 
interprétation est souvent difficile. En effet, l'activité électrique corticale du nourrisson et du jeune enfant présente des caractères la différenciant fortement de celle de l'adulte. On constate tout d'abord une extraordinaire labilité de cette activité avec l'âge et en outre de fortes différences individuelles à développement physique et intellectuel égal. Il suffira de dire que la présence de rythmes lents des types théta et delta (de 2 à 7 cycles/ seconde) auxquels on accorde le plus souvent une valeur pathologique chez l'adulte, sont des composantes normales du tracé de l'enfant à certains âges. En outre, les différents rythmes n'ont pas forcément d'un sujet à un autre ni la même amplitude, ni la même morphologie, ni la même importance relative.

Certains auteurs se sont évidemment attachés à ce problème et ont tenté de décrire et de systématiser l'évolution de l'électroencéphalogramme au cours du développement. Je citerai principalement les travaux de Smith ${ }^{13}$, de Henry ${ }^{5}$, des Gibbs ${ }^{4}$, de Hưghes et coll. ${ }^{6}$ et de H. Jasper ${ }^{7}$. Malgré l'importance et la valeur des documents rassemblés, les intéressantes conclusions auxquelles sont parvenus ces auteurs, l'interprétation de l'EEG chez l'enfant soulève encore des difficultés importantes que résolvent plus l'intuition et l'habitude de l'électroencéphalographiste, son "flair" en quelque sorte (ce qui, on en conviendra, est bien peu objectif) plutôt que des règles précises et scientifiquement établies.

Il ressort de ce que nous venons de dire, que toute aide qui nous sera donnée pour faciliter l'interprétation de l'EEG de l'enfant ne saurait être que la bienvenue. En particulier on pourrait souhaiter que les épreuves d'activation (c'est à dire des épreuves physiologiques ou pharmacologiques ayant pour effet d'accentuer ou de faire apparaître des altérations évidentes du tracé) permettent d'établir des limites tranchées entre le pathologique et le normal. Deux de cel'es-ci sont devenues de pratique courant, de "routine", dans l'examen EEG de l'adulte: l'hyperpnée et la stimulation lumineuse intermittente (S.L.I.) seule ou combinée avec l'injection de substances convulsivantes (détermination des seuils photocardiazoliques), méthode dont Gastaut a montré tout l'intérêt ${ }^{2}$.

L'hyperpnée peut être évidemment employée chez l'enfant à partir d'un certain âge, quand le sujet est capable d'obéir et de comprendre la manœuvre à éxécuter. Dans les cas les plus favorables, ce n'est guère qu'à partir de l'âge de cinq ans qu'elle est possible. En outre, elle est très difficilement réglable. Même chez l'adulte, son rythme et sa profondeur sont très difficiles à contrôler, et la multiplicité des moyens proposés pour la standardiser sont la preuve même qu'aucun d'eux n'est parfait. Ces difficultés de standardisation sont évidemment considérablement accrues chez l'enfant, sujet indocile par définition. En outre, on sait que l'hyperpnée fait apparaître des rythmes habituellement considérés comme pathologiques, chez l'enfant normal, au dessous d'un certain âge. La réactivité à cette épreuve semble en rapport surtout avec l'immaturation de l'ensemble des structures nerveuses et il est souvent très difficile devant une hyperpnée franchement 
positive, de dire s'il s'agit d'une réaction normale ou anormale compte tenu de l'âge de l'enfant.

La S.L.I. présente, au contraire, une objectivité et un intérêt beaucoup plus grands comme méthode d'activation (Walter et colls. ${ }^{14}$ ). Ses conditions optimums d'emploi (intensité, fréquence, couleur des éclairs, durée de l'épreuve) sont faciles à définir et peuvent être parfaitement standardisées. Nous allons voir d'autre part qu'à la différence de ce qui se passe chez l'adulte, l'enfant y répond avec une fréquence qui n'est pas négligeable, ceci même en dehors des cas d'épilepsie photique qui sont chez lui très rares.

Nous avons nous même l'expérience personnelle de 5.000 tracés représentant 3.348 enfants des âges les plus divers, s'étalant de quelques jours à 15 ans et pour lesquels l'épreuve d'activation par la S.L.I. a été systématiquement pratiquée. L'appareil utilisé fournit des éclairs blanc bleuté d'une intensité de 0,3 joules et d'une durée de 50 micro-secondes. La lampe est placée à 30 centimètres du sujet de façon à éclairer en plein les yeux de celui-ci. La pièce est plongée dans une demi obscurité. L'épreuve d'activation proprement dite consiste à soumettre le malade à des fréquences d'éclairement progressivement croissantes de 3 à 22 éclairs par seconde en montant très régulièrement et en restant 15 à 20 secondes sur chaque fréquence. Puis on procède de même en diminuant les fréquences d'illumination de 22 à 3 éclairs/sec. Dans le cas évidemment où une fréquence donnée s'est révélée particulièrement efficace, on y revient par la suite pour préciser l'aspect, la nature et le siège des altérations qu'elle provoque.

Nous n'avons utilisé qu'exceptionnellement les doubles éclairs suivant la technique de Gastaut (couples d'éclairs se répétant 3 fois par seconde, chaque éclair étant séparé de l'autre à l'intérieur du couple par un intervalle allant de 50 à 80 millisecondes); ceux-ci sont en effet beaucoup moins efficaces que les éclairs simples. En outre, l'usage combiné d'une substance, activante (cardiazol par exemple) ne nous est pas apparu utile ni même souhaitable. Il a été en effet démontré (Neksorocheff ${ }^{12}$ ) que les seuils photocardiazoliques déterminés suivant la méthode de Gastaut ne présentent chez l'enfant normal aucune stabilité d'un sujet à un autre de même âge, et ne sauraient donc être utlisés. En outre, l'injection intraveineuse d'une substance qui n'est pas totalement inoffensive, ne saurait être pratiquée chez de jeunes patients en mauvais état physique ou neurologique qu'avec la plus extrème réserve et dans un tout petit nombre de cas.

Quelques remarques au sujet de la technique que nous employons nous apparaissent nécéssaires. Tout d'abord, la succession des fréquences d'éclairement progressivement croissantes puis décroissantes est essentielle. Il est en effet d'observation courante que des sujets ne répondant pas particulièrement à la S.L.I. pendant que l'on augmente la fréquence des éclairs, montrent une brusque activation du tracé lorsqu'on revient en arrière, ralentissant alors le rythme des illuminations.

Le fait que l'enfant ait les yeux fermés ou ouverts pendant l'épreuve ne nous est pas apparue jusqu'à 3 ans modifier sensiblement les réponses. A partir de cet âge, les enfants acceptent de fermer volontairement les paupières et l'examen a alors toujours lieu ainsi. En outre, un avantage de 
cette épreuve qui n'est pas négligeable tient à ce fait que très souvent elle amène une réelle détente voire un calme complet chez les enfants agités ou instables qui y sont soumis, à l'inverse de ce qui se passe pour l'adulte où elle est souvent assez mal supportée. A cet effet calmant, il y a d'ailleurs parfois un léger inconvénient. La S.L.I., en particulier chez des patients de 18 mois à 3 ans, amène un assoupissement qui se traduit sur le tracé par l'apparition quasi instantanée de bouffées de rythme délta du sommeil, amples et régulières de $3 \mathrm{c} / \mathrm{sec}$. bien caractéristiques, n'ayant aucune signification pathologique. Il faut, bien entendu, veiller à ne pas les confondre avec des grapho-éléments anormaux. L'observation attentive de l'enfant durant l'examen et la connaissance de cette particularité permettent d'ailleurs une facile discrimination.

Avant de passer aux exemples destinés à illustrer l'intérêt de cette épreuve d'activation, il nous restera à définir ce que nous appellons une réponse positive à la stimulation lumineuse intermittente. Nous ne considérons comme efficaces que les épreuves faisant apparaître soit des altérations dans un tracé qui était jusqu'alors normal et pour lequel l'hyperpnée ne produisait pas de modifications pathologiques, soit amenant la production d'altérations de type très différent de celles apparues spontanément ou au cours de l'hyperpnée. Par exemple: apparition de bouffées de pointesondes typiques au cours de la S.L.I. alors qu'on ne rencontrait jusque là que quelques ondes lentes dispersées. Nous avons en revanche systématiquement négligé les examens dans lesquels la S.L.I. se contentait d'accentuer des altérations d'un type existant déjà spontanément.

En utilisant ces critères, nous avons pu constater que 146 malades avaient présenté une réponse très positive à la S.L.I., réponse dont la signification pathologique et la valeur diagnostique, pronostique ou évolutive, ne saurait être mise en doute. Pour ces 146 malades, les diagnostics cliniques se répartissaient comme suit: grand mal comitial, 10,6\%; petit mal et équivalents, 9,25\%; crises convulsives hyperthermiques ou d'origine inconnue, $22,7 \%$; retard psycho-moteur ou seulement intellectuel, $12 \%$; troubles du caractère, $18,5 \%$; pertes de connaissance, $6,35 \%$; encéphalites et chorée de Sydenham, $7 \%$; primo-infection et méningo-encéphalite tuberculeuse, $6,35 \%$; divers, $7,2 \%$.

On peut constater qu'en dehors des formes cliniques diverses de l'épilepsie qui d'ailleurs ne représentent qu'un peu moins de $20 \%$ au total, ce sont les crises convulsives de l'enfance dites hyperpyrétiques ou d'origine indéterminée, les retards de développement et les troubles du caractère qui fournissent les plus gros contingents de réponse positive à la S.L.I. $(+$ de $53 \%$ ). Ces seules constatations font déjà ressortir la valeur de cette épreuve d'activation. En effet, la pratique de l'EEG infantile montre que c'est dans ces divers domaines plus peut être que dans la comitialité proprement dite où la seule clinique impose bien souvent le diagnostic que cet examen fournit les plus intéressantes précisions. Il peut permettre en effet dans les crises convulsives de l'enfance de séparer ce qui est très probablement comitial de ce qui ne l'est presque sûrement pas, de révéler les retards psycho-mo- 
teurs et les troubles du caractère dont l'origine se trouve dans une hyperexcitabilité du systeme nerveux central et ainsi de guider la thérapeutique.

Or, ce sont dans ces différentes affections que la S.L.I. apporte le plus de renseignements. En effet, comme nous allons le voir par quelques exemples, elle est susceptible de réveler des altérations que l'activité électrique corticale du sujet au repos pas plus qu'éventuellement l'hypernée ne laissaient soupçonner.

Prenons par exemple, le cas d'une de ces crises convulsives de l'enfance dont l'origine est très difficile, pour ne pas dire impossible, à établir et le pronostic éloigné très incertain.

Notre première figure illustre le cas d'une enfant de 3 ans qui a déjà fait à deux reprises une petite crise convulsive généralisée surtout toniques, de courtes durées accompagnées d'une légère poussée fébrile ne dépassant pas $38,5^{\circ}$, elévation de température trop faible pour que l'on puisse admettre qu'il s'agit de véritables convulsions hyperthermiques. En outre, les parents auraient remarqué que leur enfant paraît parfois lointaine, "absente", observation pour laquelle il est difficile de faire la part exacte du pathologique chez un sujet aussi jeune. À ce seul rappel des anamestiques on voit immédiatement combien il est délicat de porter un diagnostic clinique exact et surtout un pronostic, ce qui dans le cas présent est essentiel.
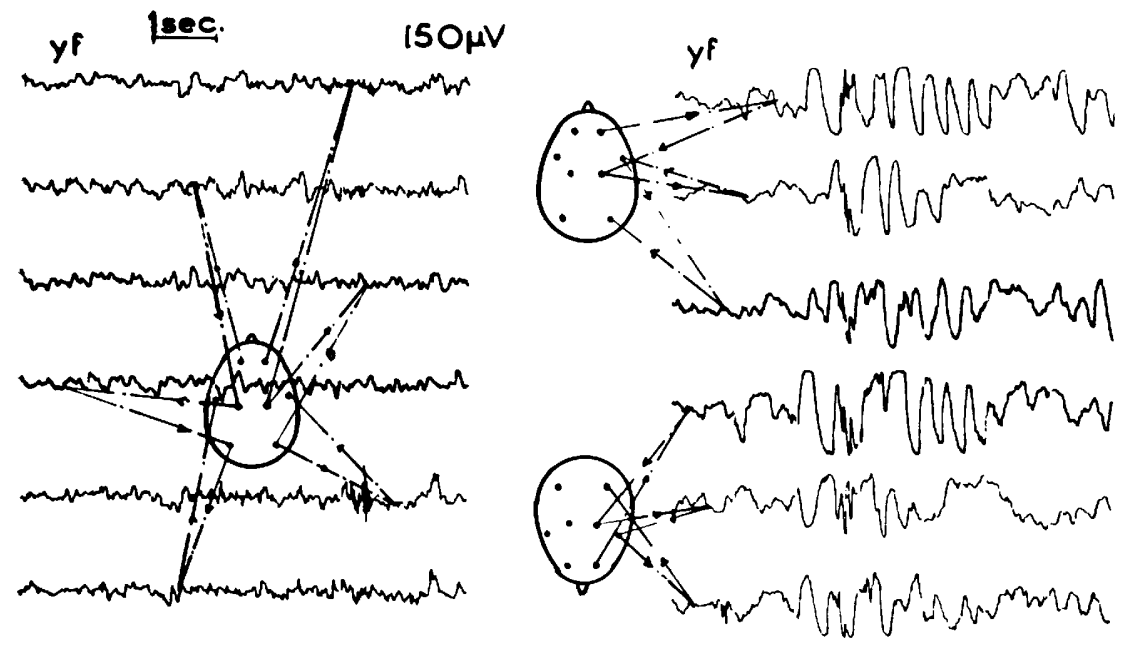

$17 \dot{e} / \sec$

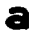

\section{b}

Fig. 1 - Enregistrement de l'activité électrique corticale spontanée (en a) et au cours de la stimulation lumineuse intermittente (en b) chez un enfant de 3 ans ayant présenté deux crises convulsives courtes et peu intenses 15 jours avant l'enregistrement. On notera l'importance des altérations que fait apparaître la S.L.I. alors qu'auparavant le tracé était normai. 
Un examen électro-encéphalographique est demandé et fait 15 jours après la dcrnière crise. Comme le montre le fragment $a$ du tracé, l'activité électrique corticale enregistrée les yeux fermés ne révèle rien de particulier et le rythme de fond apparaît très sensiblement normal au point de vue fréquence et amplitude, étant donné l'âge de l'enfant. Il n'est évidemment pas question de lui faire exécuter une hyperpnée. Én présence d'un tel résultat, on ne peut s'empêcher de trouver que l'examen n'a pas fourni tous les renseignements qu'il serait susceptible de donner. C'est dans de telles circonstances que la stimulation lumineuse intermittente offre des indications d'une valeur inappréciable. Celle-ci, pour une fréquence d'éclairement de 17è/sec., fait en effet apparaître des graphoéléments pathologiques typiques: ondes lentes à $3 \mathrm{è} / \mathrm{sec}$. de haut roltage, paroxystiques, combinées avec des éléments pointus et amples extrémement anormaux. Il apparaît évident alors qu'il existe chez notre jeune malade certains troubles des mécanismes de l'électro-génèse qui vont orienter le diagnostic vers la possibilité d'une comitialité dont les manifestations cliniques relatées sont les premiers signes. L'observation "d'absences" faite par les parents prend toute sa valeur, la thérapeutique s'oriente immédiatement un peu paradoxal en apparence chez ce petit malade bradypsychique et peu accidents convulsifs.

Les retards de développement psycho-moteurs chez les très jeunes enfants et les retards seulement intellectuels chez les enfants plus âgés posent bien souvent de redoutables problèmes à la fois diagnostiques, pronostiques et thérapeutiques. Nous n'envisageons pas évidemment ici les cas extrèmes déjà isolés et classés (myxœdème congenital, mongolisme, gargoylisme, encéphalopathies avérées), mais des formes plus légères où le ralentissement intellectuel se manifeste principalement par un retard net dans la marche, le développement du langage ou seulement une arriération scolaire très marquée.

Dans cette catégorie l'examen E.E.G. en révélant des altérations de type comitial peut rendre d'inappréciables services. La figure 2 illustre un de ces cas où, chez un enfant de 12 ans et demi présentant un important retard intellectuel, l'E.E.G. sans activation non plus que l'hyperpnée ne révèlent rien de particulier si ce n'est une pauvreté certaine des rythmes normaux, en particulier de l'alpha qui est peu net, de bas voltage et assez irrégulier, mais de fréquence normale. Ce sont là des indices bien insuffisants pour orienter vers l'étiologie ou a fortiori la thérapeutique. Enn revanche, la S.L.I. fait apparaitre pour des fréquences variées d'illumination de 12 à $18 \grave{e ̀} / \mathrm{sec}$. et en particulier pour $15 \grave{\mathrm{e}} / \mathrm{sec}$. des altérations manifestes de type paroxystique: pointes, pointes-ondes, ondes lentes qui traduisent une instabilité importante des mécanismes de l'électrogénèse. Cette constatation amène à préconiser et à instituer une thérapeutique calmante à base de barbituriques, traitement un peu paradoxal en apparense chez ce petit malade bradypsychique et peu actif, mais qui a donné d'excellents résultats et amené une très nette amélioration.

Les troubles du caractère constituent également chez l'enfant une entité clinique bien vaste et il est fort difficile bien souvent de déméler ce qui relève de la seule psychothérapie, des cas peut-être plus organiques et justiciables d'une thérapeutique "pharmacologique". Dans ce domaine encore, la pratique de l'EEG, surtout lorsqu'elle est complétée par l'activation photique amène parfois à d'intéressantes constatations. C'est ainsi que la figure 3 met en lumière l'une d'entre elles.

Il s'agit là d'un petit garçon de 6 ans et demi, dont l'instabilité de caractère, l'agitation incessante, les tics nombreux et variés ont fini par inquiéter la famille. 

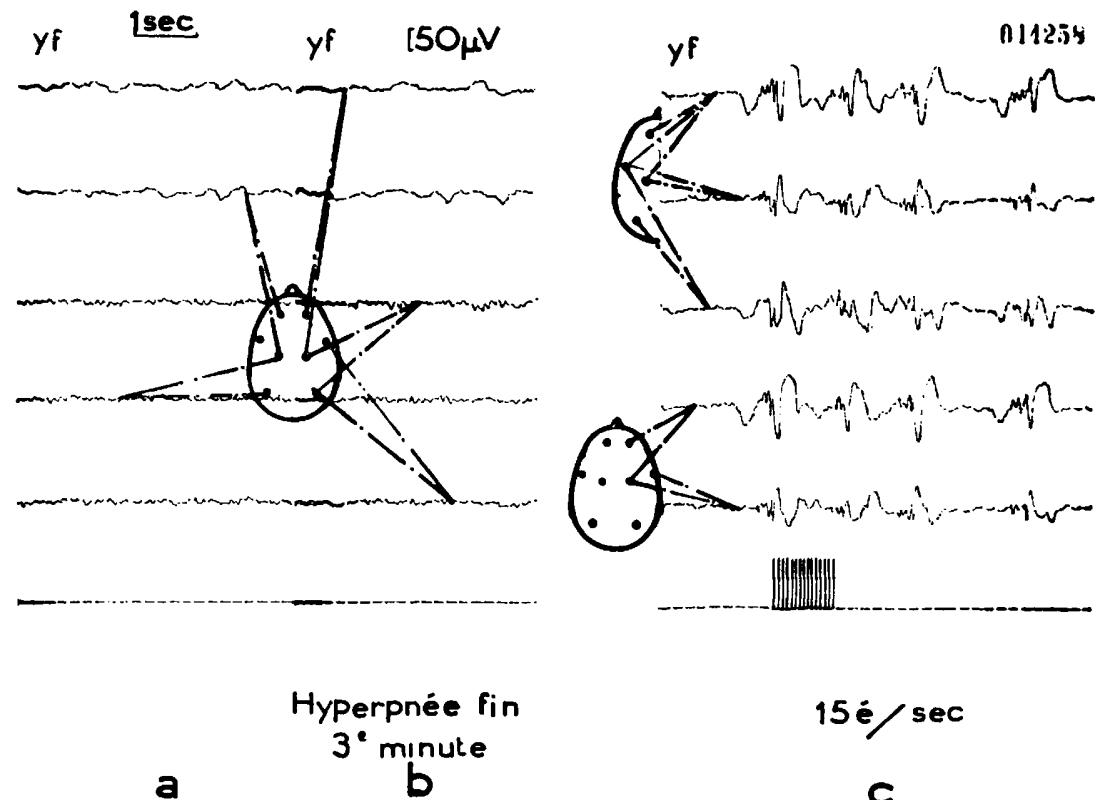

a

Fig, 2 - Electro-encéphalogramme d'un enfant de 12 ans ei demi présentant un important retard scolaire. L'activité électrique spontanée est sensiblement normaie, l'hyperpnée après trois minutes sans effets activants. La S.L.I. provoque l'apparition d'éléments paroxystiques caractéristiques (pointes-ondes). La ligne inférieure du tracé a été réservée au marquage des tops corresponciant aux éclairs du photo-stimulateur.

L'aspect purement psychiatrique des troubles n'apparaissant pas l'élément le plus intéressant au médécin consulté, celui-ci demande ụ électro-encéphalogramme.

Le tracé standard ne montre rien de particulier seulement un bas voltage généralisé de l'alpha qui est peu net et assez instable, mais de fréquence normale compte tenu de l'âge de l'enfant. Il n'y a pas trace d'éléments paroxystiques et l'hyperpnée ne modifie pas sensiblement l'aspect de l'enregistrement alors qu'elle a été très convenablement effectuée. En revanche la S.L.I. provoque l'apparition d'éléments paroxystiques typiques (polypointes, ondes) accompagnées même, dans ce cas de secousses myocloniques pour des fréquences d'éclairement de 10 à 20 éclairs/sec., mynclonies faciales d'abord qui ont ensuite gagné les membres supérieurs.

Plus curieux encore est le cas illustré par la figure suivante (fig. 4). Il s'agit là d'une fillette de 14 ans dont les troubles, d'une grande banalité, consistaient en hémicéphalées gauches avec des colères impulsives violentes et ceci depuis la petite enfance et chez qui l'E.E.G. standard non plus que l'activation par l'hyperpnée ne font apparaitre aucune altération alors qu'à la fin de l'épreuve de S.L.I., dans la phase des fréquences d'éclairemient décroissantes, se produisent des altérations de type comitial indiscutable accompagnées d'une crise bravais-jacksonienne typique touchant seulement le membre supérieur droit. Premier et d'ailleurs unique accident convulsif mettant en évidence l'instabilité et l'hyperexcitabilité centrale de ce sujet et probablement l'indice d'une micro-lésion localisée à l'hémisphère gauche et que les seuls signes cliniques ne permettaient pas jusque là de soupçonner. 


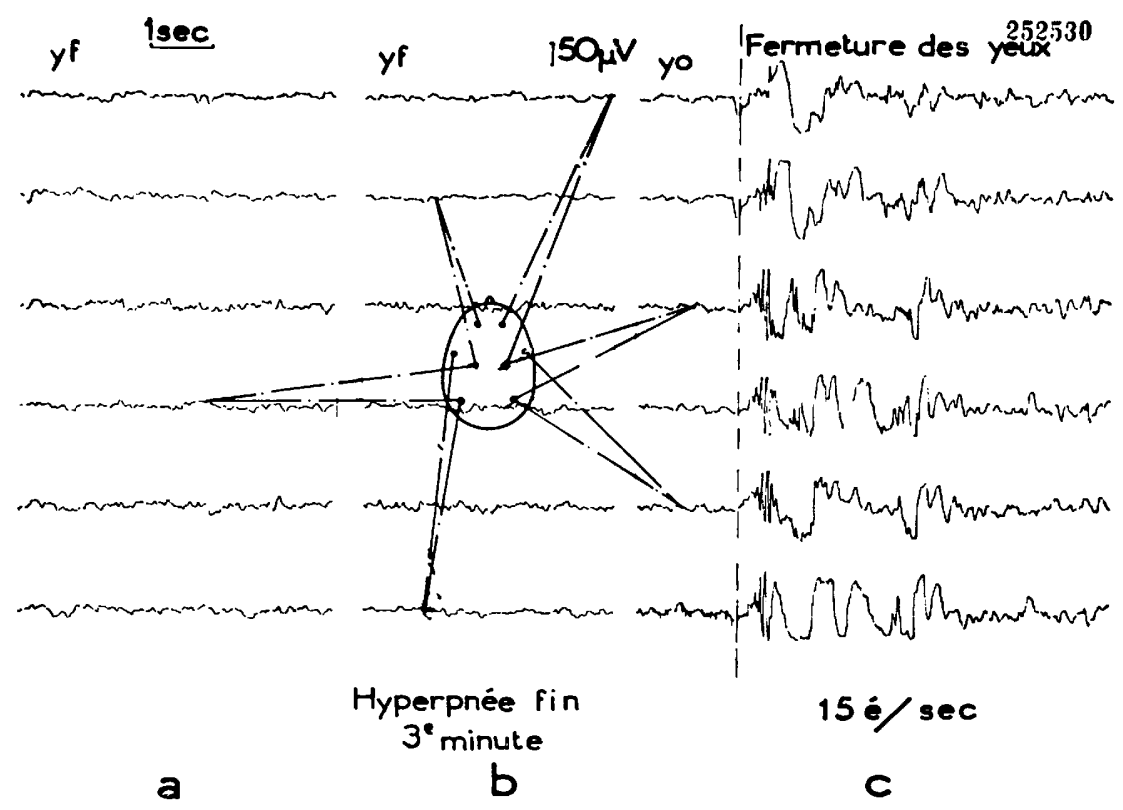

Fig. 3 -- Tracé électro-encéphalographique standarō (en a), à la fin de la 3 ème minute d'hyperpnée (en b) d'un enfant de 6 ans et demi présentant des tics et de l'instabilité. Noter l'intense pouvoir activant de la stimulation lumineuse intermittente (en c).

L'exposé des différents cas que nous venons d'étudier nous paraît avoir bien mis en évidence l'intérêt de la stimulation lumineuse intermittente comme procédé d'activation dans l'EEG de l'enfant, mais on ne saurait dire actuellement avec exactitude sur quels méchanisms physiologiques ou plus exactement physio-pathologiques elle repose. De nombreux travaux ont déjà été consacrés aux effets chez l'adulte de la S.L.I. combinée à l'action de substances convulsivantes, en particulier le cardiazol. On s'accorde à peu près pour admettre que les substances convulsivantes abaissent les seuils synaptiques des systèmes de projection entre le cortex occipital et certains noyaux thalamiques d'une part, et entre ceux-ci et l'ensemble des projections thalamo-corticales d'autre part, ce qui amène l'apparition du fonctionnement synchrone anormal de certains groupes cellulaires (Jasper ${ }^{7}$ ) d'où apparition de complexes pointes-ondes, à partir de l'excitation du cortex occipital par les éclairs lumineux rythmés (Gastaut ${ }^{3}$, Jasper ${ }^{8}$, Lindsley, Bowden et Magoun ${ }^{10}$, Moruzzi et Magoun ${ }^{11}$ ).

Certains auteurs (Gastaut ${ }^{3}$ ) ont même pensé pouvoir décrire une véritable courbe d'état d'excitation des systèmes neuro-synaptiques responsables de la réponse photomyoclonique par "recrutement" et considéré que, de ce fait, seule une étroit bande de fréquence autour de 15 éclairs/sec. était susceptible de faire apparaître celle-ci. 


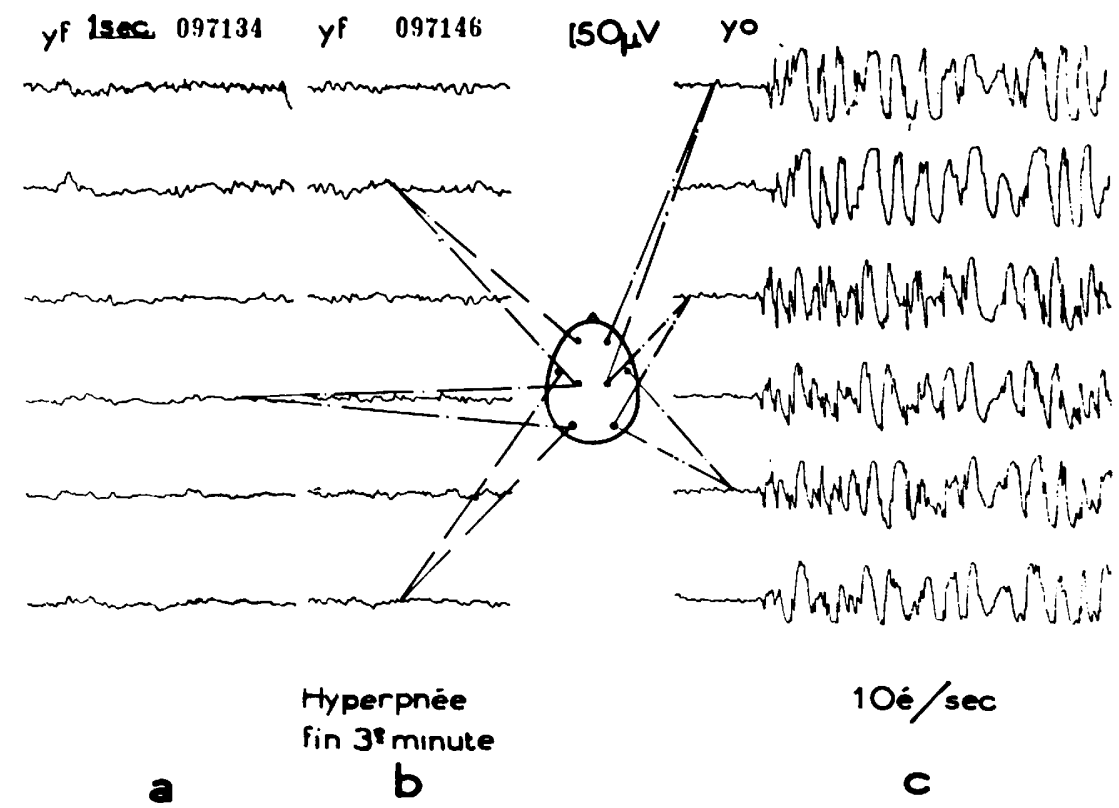

Fig. 4 - Electro-encéphalogramme standard (en a), après 3 minutes d'hyperpnée (en b) et au cours de la S.L.I. à 10 éclairs/s. (en c) d'une fillette de 14 ans présentant des hémi-céphalées et des colères violentes. Une crise BJ droite se déclanche en même temps qu'apparaîssent les altérations à la S.L.l. Noter que celles-ci naissent alors que l'enfant a les yeux ouverts.

Pour l'enfant le problème se pose différemment. En effet, chez lui, le phénomène apparaît fort complexe. Nous avons pour notre part étudié seulement les réponses à la S.L.I. à l'exclusion de sa combinaison avec l'action d'une substance convulsivante pour des raisons de sécurité faciles à comprendre. Malgré cette différence important, il nous a été possible de constater que cette technique était capable de provoquer l'apparition d'altérations paroxystiques typiques voire de déclencher des myoclonies. L'interprétation du phénomène reste délicate mais quelques faits nous paraissent devoir être mentionnés. Tout d'abord chez l'enfant, il se révèle indispensable de faire durer l'épreuve suffisamment car une très forte proportion de cas, les graphoéléments pathologiques n'apparaissent que lorsque l'on est dans la phase des fréquences décroissantes d'éclairement. C'est ainsi que pour les 146 malades envisagés, $16,5 \%$ présentaient une réponse nette ou maximum au cours de la seule phase d'augmentation des fréquences d'éclairement, tandis que $63,5 \%$ présentaient une réponse à la photostimulation seulement au cours de la phase de diminution de fréquence $(30 \%)$ ou maximum pendant celle-ci $(33,5 \%)$. On peut comprendre ce fait en admettant qu'indépendamment des questions de facilitation temporelle au stade élémentaire des systèmes synaptiques, la S.L.I. quand elle dure suffisamment produit une sorte de sensibilisation générale des méchanismes de 
l'électro-génèse abaissant le seuil de réponse paroxystique un peu comme le fait l'injection d'une substance convulsivante, type cardiazol qui imprègne petit à petit tout le système nerveux central.

On constate d'autre part chez les enfants qu'en général les réponses paroxystiques apparaissent pour des gammes de fréquence d'éclairement assez larges, fait qui s'oppose à l'acceptation de méchanismes aussi simples que celui proposé par Gastaut.

Enfin, et ce sera la notre conclusion, la signification et la valeur de la S.L.I. en tant qu'épreuve d'activation destinée à mettre en évidence certaines anomalies des structures responsables de d'électrogénèse, nous paraît démontrée par cette seule observation de deux jumeaux univitellins, âgés de 8 ans, présentant tous deux des troubles du caractère et chez qui la S.L.I. amenait la production de polypointes-ondes accompagnées de myoclonies de la face et des membres pour des fréquences d'éclairement strictement égales (12⿺̀丶/sec.). L'épreuve d'activation reflétait ainsi la parité si complète des structures nerveuses que l'on sait exister dans ce cas.

\section{RÉSUMÉ}

L'électroencéphalographie de l'enfant, surtout lorsqu'il est très jeune, présente de sérieuses difficultés, à la fois de technique et d'interprétation. Les épreuves d'activation sont, en conséquence, de grande utilité. Une de celles-ci nous paraît de tout particulier intérêt: il s'agit de la stimulation lumineuse intermittente seule, sans injection de substances convulsivantes.

Utilisée systématiquement suivant certaines règles précises, elle a permis de révéler dans un important pourcentage de cas, des altérations de type paroxystique apportant ainsi une aide précieuse au diagnostic, principalement dans les crises convulsives de l'enfance, les retards psychomoteurs et les troubles du caractère. Quelques exemples empruntés à ces différentes catégories d'affections font ressortir le pouvoir activant de la photostimulation.

Les méchanismes de ce pouvoir sont très délicats à interpréter et font intervenir probablement à la fois l'immaturation des structures thalamocorticales existant chez l'enfant et les méchanismes neurophysiologiques déjà connus de l'activation photocardiazolique étudiée chez l'adulte. Quoiqu'il en soit, cette méthode apparaît du plus haut intérêt dans la pratique de l'électroencéphalographie de l'enfant.

\section{BIBLIOCRAPHIE}

1. Ellington, R. J.; Lindsley, D. 13. - Brain waves and cortical development in newborn and young infants. The Am. Psychologist., 4:248, 1949. 2. Gastaut, H. - Combined Photic and Metrazol activation of the Brain. E.G.-Clin. Neurophysiol, 2:249, 1950. 3. Gastaut, H.; Corriol, J.; Roger, A. - Le cycle d'excitabilité des systèmes afférents corticaux che\% l'homme. Rev. Neurol., 84:602, 1951. 
4. Gibbs, F. A.; Gibbs, L. L. - Atlas of Electroencephalography. Cummings Ed. Cambridge (Mass.) U.S.A., 1941. 5. Henry, C. E. - Electroencephalograms of normal children, Soc. Res. in Child Development. N.R.C., 9.3. Serial 39, 1944. 6. Hughes, J. G.; Ehemann, B.; Brown, U. - EEG of the newborn. Ann. J. Dis. Child., 76:503, 1949 e 77:310, 1949. 7. Jasper, H. - - Diffuse projections systems: the integrative action of the thalamic reticular system. F.EG Clin. Neurophysiol., 1:405, 1949. 8. Jasper, $\mathrm{H}$. - Effect of thalamic stimulation in unaesthized animals. EEG Clin. Neurophysiol., 1:305, 1949. 9. Jasper, H. - EEG in child Neurology and Psychiatry. Pediatrics, 3:783, 1949. 10. Lindsley, D. B.; Bowden, J. W.; Magoun, H. W. - Effect upon the EEG of acute injury to the brain stem activating system. EEG Clin. Neurophysiol., 1:491, 1949. 11. Moruzzi, G.; Magoun, H. W. - Brain stem reticular formation and activation of the EEG. EEG Clin. Neurophysiol., 1:455, 1949. 12. Nekhorocheff, I. -- Seuil photo-cardiazolique chez l'enfant. Rev. Neurol., 83:599, 1950. 13. Smith, J. R. - 'The EEG during normal infancy and childhood. J. Genit. Psychol., 1938, 53:431-455-471, 1938.14. Walter, W.; Grey-Rovey, V. J.; Shipton, H. - Analysis of the electrical response of the human cortex to photic stimulation. Nature, 158:540, 1948.

Rue J'ictor Cousin, 1 - Paris, France. 